\title{
Roots Blower with Gradually Expanding Outlet Gap: Mathematical Modelling and Performance Simulation
}

\author{
Yingjie Cai ${ }^{1, a}$, Ligang $\mathrm{Yao}^{2, \mathrm{~b}}$ \\ ${ }^{1}$ School of Mechanical Engineering and Automation, Fuzhou University, Fuzhou, 350116, China \\ ${ }^{2}$ School of Mechanical Engineering and Automation, Fuzhou University, Fuzhou, 350116, China \\ aemail: cyj@fzu.edu.cn, bemail: ylgyao@fzu.edu.cn
}

Keywords: Gradually Expanding Gap; Roots blower; Basic inter-space; Simulation

\begin{abstract}
Gradually expanding gap in the outlet of the Roots blower can effectively reduce the backflow impact and the noise of the blower. The working principle for the Roots blower with the gradually expanding gap is investigated in this paper. And the mathematical modelling for backflow is proposed. Numerical methods are given to solve the outlet gap size. The performance parameters of different dimension gap are compared. The outlet flow field in Roots blower with gradually expanding gap is simulated by means of CFD simulation. Finally, the correctness of the numerical solution is verified.
\end{abstract}

\section{Introduction}

The Roots blower with the characteristics of large range of output pressure, higher speed, and large exhaust air rate has been used widely [1]. As shown in Fig.1, two rotors are driven by a pair of synchronous gears rotating in opposite direction at the same speed. While in working process, a closed space is formed between the rotor profile and the inner walls of the housing, and the closed space is called the basic inter-space. With the rotation of a pair of rotors, gas is pushed from the inlet to the outlet in the basic inter-spaces. Without taking into account the leakages, there are 6 basic inter-spaces of the gas which are delivered with a revolution of the rotors. Because of the large pressure differentials between the inlet and the outlet gas, the high pressure gas of the outlet will have a greater impact when the basic inter-space is transferred to the outlet. In addition, the trapped gas when the rotors engaging can cause the local pressure to rise rapidly. And all the impact will produce vibrations and noises. Roots blower noise reduction has been a research focus of scholars. Xiong put the muffler into the Roots blower shell, and reduced the noise by $15 \sim 25 \mathrm{~dB}$ [2]. $\mathrm{Li}$ studied the countercurrent cooling structure and gradually expanding gap of Roots blower, and verified the effectiveness of them with theoretical analysis and numerical simulation [3]. Wang analyzed the vibration energy of Roots blower equipments and noise propagation both qualitatively and quantitatively with the spectral characteristics of vibration and wideband noise taken into account [4]。Fu solved the gradually expanding gap of Roots blower by curve fitting method [5].

Many studies show that the gradually expanding gap of the Roots blower is one of the effective methods reducing noises. But there is no calculation method for the expanding gap. The gradually expanding gap of Roots blower is analyzed, and the gas flow equation is established in this paper. Each of linear and quadratic function is chosen as the changing rule of the gradually expanding gap. The backflow process is modeled by mathematics. The optimal exit size of the gradually expanding gap is solved with numerical method. Finally, the Roots blower outlet flow field is simulated by CFD software. And the correctness of the numerical solution is verified.

\section{The Theoretical Analysis and Mathematical Modeling of the Gradually Expanding Gap}

One of the effective ways to reduce the backflow impact is to use the helical rotors similar to the helical gear. The basic inter-space in the Roots blower with helical rotors is gradually opened at the outlet, which can effectively reduce the impact of the backflow at the outlet. However, due to the characteristics of the structure, the manufacturing of the helical rotors is difficult, and the 
production cost is higher. So, it is rarely used in practical Roots blower production. And another feasible but more economical means to reduce the impact is to use the outlet gradually expanding gap [3]. As shown in Fig. 2, the inner housing wall near the outlet of the blower is gradually expanding; a wedge-shaped space between the outer circle of the rotor tooth profile and the wall of the blower housing is formed. So, the gap between the rotors and the walls is gradually increasing with the rotation of the rotors. The pressure of basic inter-space is gradually increasing when the backflow of the gas through the gap which are gradually increasing. When the basic inter-space connects with the outlet space of the blower, the pressure of the basic inter-space and the outlet space gets to balance. The gradually expanding gap of the blower can effectively reduce the impact of the backflow and the noise of the blower.
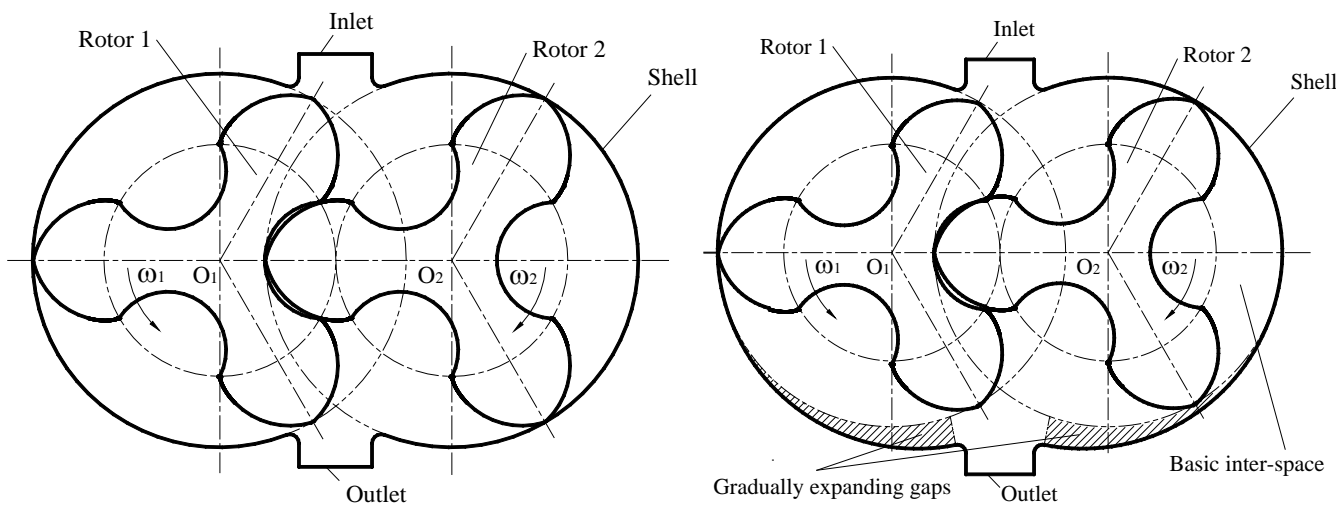

Fig.1 Roots blower

Fig. 2 The gradually expanding gaps of the outlet

In order to avoid the collision between the two rotors, between the rotors and the inner housing wall, the gap is set between them. The gap between rotor and the inner housing wall can be calculated by the following formula [1],

$\delta_{r}=(0.005 \sim 0.001) D$.

Where in Eq. (1), the $\delta_{r}$ is the width of gap between the rotor and the inner housing wall; $D$ is the addendum circle diameter of the rotor tooth profile.

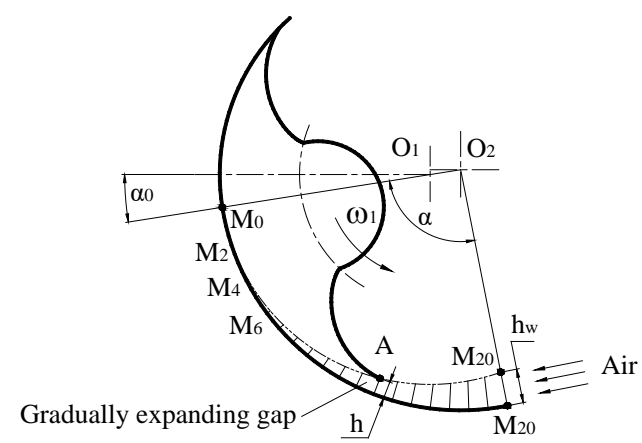

Fig. 3 The basic inter-space and the gradually expanding gap

Even if the Roots blower doesn't have gradually expanding gap, there are backflow and leakages through the gap mentioned above. But, the backflow and leakage rate is basically unchanged and smaller. So, we can neglect the gap mentioned above when solving the gradually expanding gap. As shown in Fig. 3, when the point $A$ on the rotors runs from $M_{0}$ to $M_{20}$, the gap width $h$ between the rotor tooth profile and the housing wall increases gradually from 0 to $h_{w}$. And the pressure in the basic inter-spaces increases gradually; meanwhile the pressure differentials reduce gradually. At the exit, the velocities of backflow decrease to zero, and the pressure differential is to zero too. So, the backflow impact is greatly reduced.

In engineering applications, the design of the gap value is often based on the experiences [5]. However, too small gap can't reduce the impact effectively, and too large gap will enlarge the size 
of the blower. In this paper, calculation model of the flow-back process when the basic inter-space gets through the gradually expanding gap is established. And the optimal values of the gradually expanding gap are obtained. The change law of backflow parameters is studied.

In the backflow processes, the air input of the basic inter-space is given as [6],

$$
Q=\int_{0}^{t_{20}} \rho_{w} v h d t
$$

In Eq. (2), $Q$ is the intake air quality, $t_{20}$ is the time when point $A$ gets through the gradually expanding gap, $\rho_{w}$ is the air density in outlet, $v$ is the velocity of backflow, $h$ is the gap width.

The values of $h$ are from 0 to $h_{w}$, and change by linear or quadratic function law.

$$
\begin{aligned}
& h=h(t) \\
& h=h\left(t^{2}\right)
\end{aligned}
$$

The velocities of backflow is,

$$
v=\varphi \sqrt{2\left(p_{w}-p_{n}\right) / \rho_{w}}
$$

Where in Eq. (5), $\varphi$ is the velocity coefficient, and its value is 0.64 [4]; $p_{w}$ and $p_{n}$ are pressures in the exit and basic inter-space respectively.

The gauge pressure in outlet of Roots blower is usually no more than $80 \mathrm{kPa}$, and the efficiency of the blower reaches the maximum value when gauge pressure is $40 \mathrm{kPa}$ or so. In order to simplify calculating, we build a gas model approximately by ideal gas. The air pressure in the basic inter-space will increases with the increasing of the backflow air quality.

Clapyron equation of ideal gases:

$$
p V=n R T
$$

Where in Eq. (6), $p$ represents the gas pressure; $V$ is the gas volume; $n$ is the amount of matter; $R$ represents the gas constant; $T$ is the absolute temperature.

From Eq. (6), it can be seen that in the case of constant temperature gives,

$p \propto n / V \propto \rho$

Because a basic inter-space volume of the rotor is fixed, so:

$$
p_{n}=\frac{Q_{0}+Q}{Q_{0}} p_{0}
$$

In Eq. (8), $Q_{0}$ is the initial air quality of the basic inter-spaces, $p_{0}$ is the initial air pressure of the basic inter-space.

Outlet air density:

$$
\rho_{w}=\frac{p_{w}}{p_{0}} \rho_{0}
$$

Where, $\rho_{0}$ is the air density with one standard atmospheric pressure, $h_{w}$ is solved by Eqs (2), (5) and (8). The boundary condition is given as $\Delta p=p_{w}-p_{n}=0$, when $t=t_{20}$. Because of the difficulty of solving differential equations, the equation variables are discreted to deal with.

The gradual expansion area is divided into 20 parts, as shown in Fig. 3. Let $i=1: 20$, then:

$$
\left\{\begin{array}{l}
Q_{i+1}=Q_{i}+\Delta t \cdot v_{i} \cdot h_{i} \cdot \rho_{w} \\
v_{i}=\varphi \sqrt{2\left(p_{w}-p_{n i}\right) / \rho_{w}} \\
p_{n i+1}=p_{n i}+Q_{i+1} / Q_{i} p_{n i}
\end{array}\right.
$$




\section{An Example of Solving Gradually Expanding Gap}

Assuming that center distance of two rotors is $a=127 \mathrm{~mm}$, diameter of rotor addendum circle is $D=204 \mathrm{~mm}$, the rotors profiles are designed by the eccentric arcs and cycloidal curves tooth profile [9], the initial pressure in the basic inter-space is $p_{0}=101325 \mathrm{~Pa}$, the initial angle of the gradually expanding gap area is $\alpha_{0}=9^{\circ}$, the whole angle of the gradually expanding gap area is $\alpha=100^{\circ}$, the rotor speed is $n=1460 \mathrm{rpm}$, the gauge pressure of outlet is $40 \mathrm{kPa}$, the total pressure of the outlet is $p_{w}=141325 \mathrm{~Pa}$.

The gradual expansion area is divided into 20 parts, as shown in Fig. 3. Let $i=1: 20$. According to the rotor speed and the whole angle of the gradual expansion area, the interval time can be solved and given as $\Delta t=0.000571 \mathrm{~s}$. The boundary condition is given as $\Delta p_{20}=0, v_{20}=0$. The gradually expanding gap is calculated by linear and quadratic function changing rule respectively by solving the Eq. (10), and the calculation results are shown in Tab. 1 and Tab. 2.

Tab.1. Calculation result of backflow in the gradually expanding gap with linear function changing rule

\begin{tabular}{|c|c|c|c|c|c|}
\hline No. $i$ & $\begin{array}{c}\text { Air qualities in } \\
\text { basic inter-space } \\
m_{i}(k g)\end{array}$ & $\begin{array}{c}\text { Air pressures in } \\
\text { basic inter-space } \\
p_{n i}(P a)\end{array}$ & $\begin{array}{c}\text { Pressure } \\
\text { differentials } \\
\Delta p_{i}(P a)\end{array}$ & $\begin{array}{c}\text { Velocities of } \\
\text { backflow } \\
v_{i}(m / s)\end{array}$ & $\begin{array}{c}\text { Gap widths } \\
h_{i}(m)\end{array}$ \\
\hline 1 & 0.00834 & 101325 & 40000 & 134.70 & 0.00021 \\
\hline 2 & 0.00837 & 101686 & 39639 & 134.09 & 0.00043 \\
\hline 3 & 0.00843 & 102405 & 38920 & 132.87 & 0.00064 \\
\hline 4 & 0.00852 & 103473 & 37852 & 131.03 & 0.00086 \\
\hline 5 & 0.00863 & 104877 & 36448 & 128.58 & 0.00107 \\
\hline 6 & 0.00878 & 106600 & 34725 & 125.50 & 0.00128 \\
\hline 7 & 0.00894 & 108618 & 32707 & 121.80 & 0.00150 \\
\hline 8 & 0.00913 & 110903 & 30422 & 117.47 & 0.00171 \\
\hline 9 & 0.00934 & 113421 & 27904 & 112.50 & 0.00193 \\
\hline 10 & 0.00956 & 116135 & 25190 & 106.89 & 0.00214 \\
\hline 11 & 0.00980 & 118999 & 22326 & 100.63 & 0.00235 \\
\hline 12 & 0.01004 & 121966 & 19359 & 93.71 & 0.00257 \\
\hline 13 & 0.01029 & 124979 & 16346 & 86.11 & 0.00278 \\
\hline 14 & 0.01054 & 127979 & 13346 & 77.81 & 0.00299 \\
\hline 15 & 0.01078 & 130898 & 10427 & 68.77 & 0.00321 \\
\hline 16 & 0.01100 & 133662 & 7663 & 58.96 & 0.00342 \\
\hline 17 & 0.01121 & 136190 & 5135 & 48.26 & 0.00364 \\
\hline 18 & 0.01139 & 138389 & 2936 & 36.50 & 0.00385 \\
\hline 19 & 0.01154 & 140149 & 1176 & 23.10 & 0.00406 \\
\hline 20 & 0.01163 & 141325 & 0 & 0.00 & 0.00428 \\
\hline
\end{tabular}


Tab.2. Calculation result of backflow in the gradually expanding gap with quadratic function changing rule

\begin{tabular}{cccccc}
\hline No. $i$ & $\begin{array}{c}\text { Air qualities in } \\
\text { basic inter-space } \\
m_{i}(\mathrm{~kg})\end{array}$ & $\begin{array}{c}\text { Air pressures in } \\
\text { basic inter-space } \\
p_{n i}(\mathrm{~Pa})\end{array}$ & $\begin{array}{c}\text { Pressure } \\
\text { differentials } \\
\Delta p_{i}(\mathrm{~Pa})\end{array}$ & $\begin{array}{c}\text { Velocities of } \\
\text { backflow } \\
v_{i}(\mathrm{~m} / \mathrm{s})\end{array}$ & $\begin{array}{c}\text { Gap widths } \\
h_{i}(\mathrm{~m})\end{array}$ \\
\hline 1 & 0.00834 & 101325 & 40000 & 134.70 & 0.00002 \\
\hline 2 & 0.00834 & 101352 & 39973 & 134.65 & 0.00006 \\
\hline 3 & 0.00835 & 101458 & 39867 & 134.48 & 0.00014 \\
\hline 4 & 0.00837 & 101698 & 39627 & 134.07 & 0.00025 \\
\hline 5 & 0.00841 & 102122 & 39203 & 133.35 & 0.00039 \\
\hline 6 & 0.00846 & 102781 & 38544 & 132.22 & 0.00057 \\
\hline 7 & 0.00854 & 103723 & 37602 & 130.60 & 0.00077 \\
\hline 8 & 0.00864 & 104988 & 36337 & 128.38 & 0.00101 \\
\hline 9 & 0.00878 & 106614 & 34711 & 125.48 & 0.00128 \\
\hline 10 & 0.00894 & 108624 & 32701 & 121.79 & 0.00158 \\
\hline 11 & 0.00914 & 111033 & 30292 & 117.22 & 0.00191 \\
\hline 12 & 0.00937 & 113838 & 27487 & 111.66 & 0.00227 \\
\hline 13 & 0.00963 & 117018 & 24307 & 105.00 & 0.00267 \\
\hline 14 & 0.00992 & 120528 & 20797 & 97.13 & 0.00309 \\
\hline 15 & 0.01023 & 124293 & 17032 & 87.90 & 0.00355 \\
\hline 16 & 0.01055 & 128205 & 13120 & 77.14 & 0.00404 \\
\hline 17 & 0.01088 & 132111 & 9214 & 64.65 & 0.00456 \\
\hline 18 & 0.01118 & 135806 & 5519 & 50.03 & 0.00512 \\
\hline 19 & 0.01144 & 139013 & 2312 & 32.39 & 0.00570 \\
\hline 20 & 0.01163 & 141325 & 0 & 0.00 & 0.00632 \\
\hline
\end{tabular}

From the Tab. 1 and Tab. 2, it can be seen that both pressure differentials and velocities of backflow reduce to zero when point $A$ on the rotor reaches to the exit of the gradual expansion, and the backflow is disappeared this moment. The gap width of exit of linear function is $4.28 \mathrm{~mm}$, and the quadratic function is $6.32 \mathrm{~mm}$. The linear function is better than the quadratic function. From the change trend of pressure differentials in Fig. 4, we can see that the pressure differential of the linear function showing smaller overall trend. The linear function is better than the quadratic function.

As shown in Fig. 5, the backflow velocities of linear function are lower than that of quadratic function, and change more smoothly. The linear function is better than the quadratic function.

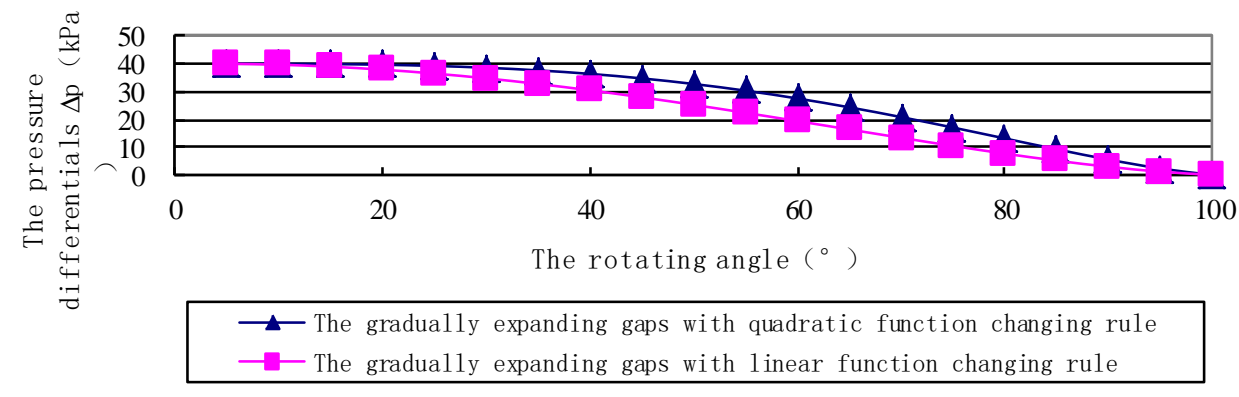

Fig.4 The pressure differentials changing curves

\section{Comparison and Verification of Roots Blower Pressure Pulsation Based on CFD}

To verify the effect of the gradually expanding gap, taking linear function changing rule gradually expanding gap of Roots blower and non-gradually expanding gap of Roots blower into compared. In CFD simulation, the unsteady flow field is used, the dynamic mesh is adopted, and the rotation speed of the rotor is given as $1460 \mathrm{rpm}$. The fluid pressure and velocity of two kinds of blower, which are in a revolution of rotor, the normal gap and the linear function changing gap, are solved. Due to limitations on space, the process of modeling and solving process is omitted.

Based on the comparison results above, the performance of Roots blower with gradually expanding gap in linear function changing rule are better than that of quadratic function. Due to 
limitations on space, the other changing rule of the gradually expanding gap isn't discussed here. Considering the convenience of design and manufacture, it is appropriate to choose the linear function rule.

Fig. 6 and Fig. 7 are the flow velocity distribution in the Roots blower at the time when the gap is rotating near to the exit. The large flow rate is in the gap between the two rotors of the backflow, this kind of local high speed backflow cannot be avoided. Near to the exit of the normal gap Roots blower has a intense backflow, as shown in Fig. 8. And the backflow velocity of the Roots blower with gradually expanding gap is obviously decreased, as shown in Fig. 9.

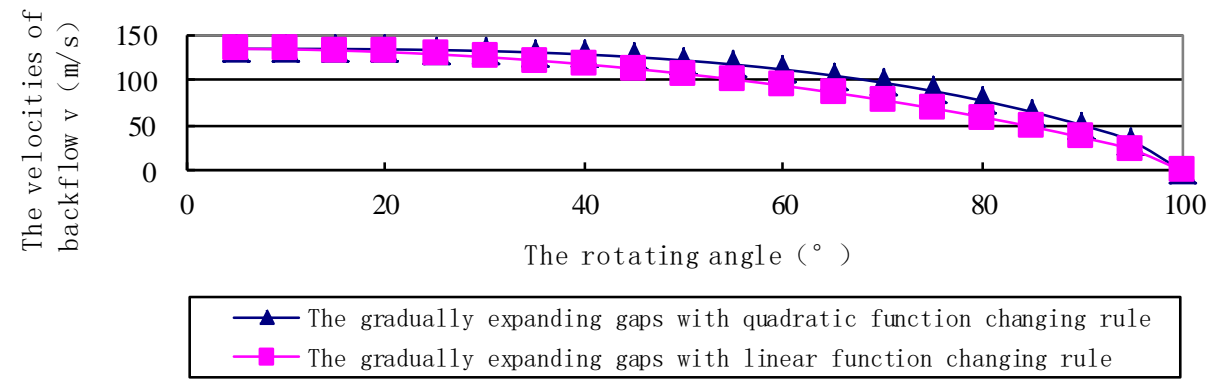

Fig.5 The velocity of backflow changing curves

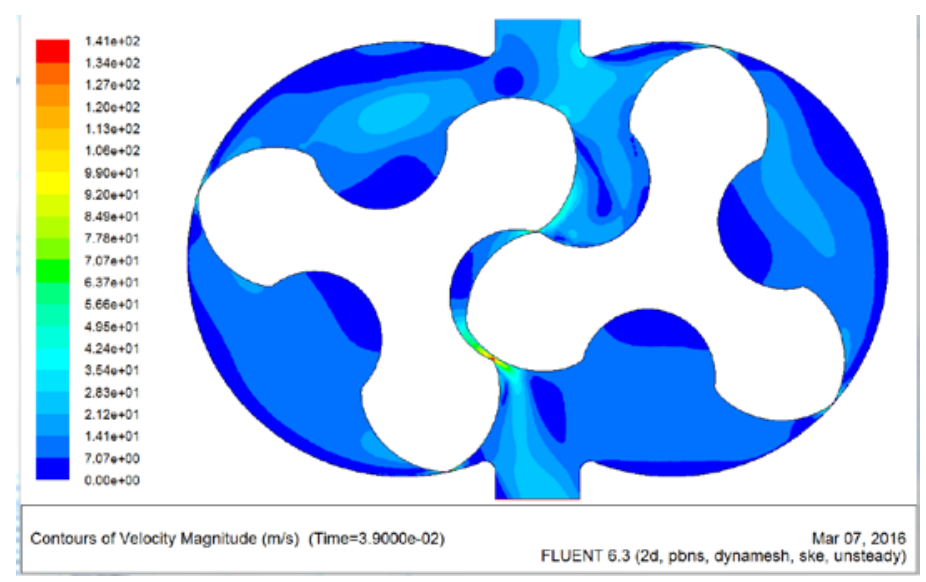

Fig.6 The flow velocity distribution in Roots blower with normal gap

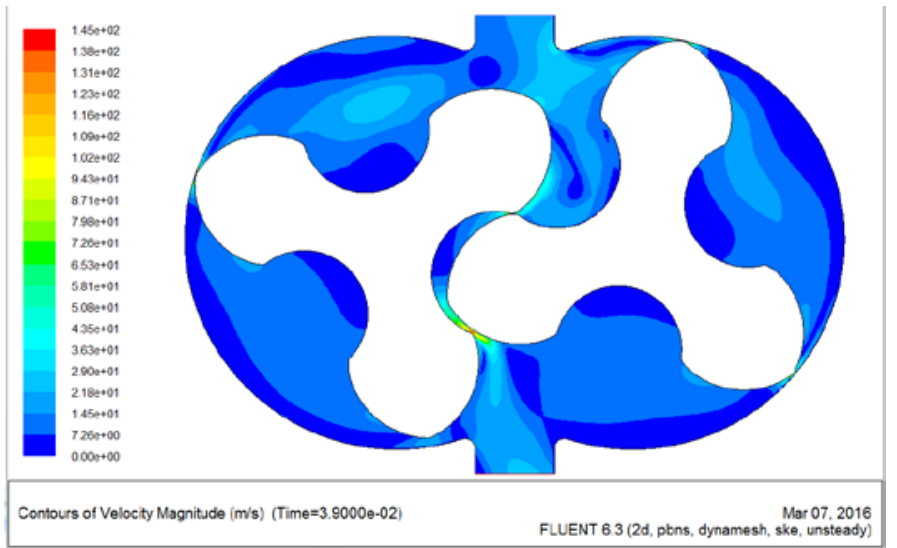

Fig.7 The flow velocity distribution in Roots blower with gradually expanding gap

From Fig. 8, we can see that the velocity of the backflow reaches to $240 \mathrm{~m} / \mathrm{s}$, and is much higher than that of the flow field nearby. When the basic inter-space opens to the exit, the high-pressure airs will impact the basic inter-space of airs intensely, accompanied by flow pulsation and noise. And the backflow velocity of the Roots blower with gradually expanding gap is only about $30 \mathrm{~m} / \mathrm{s}$, which is consistent with the theoretical calculation results, as shown in Tab. 1 . Obviously, using the gradually expanding gap can significantly slow down the backflow rate and reduce the air pulsation and noise. The effects are obviously. 


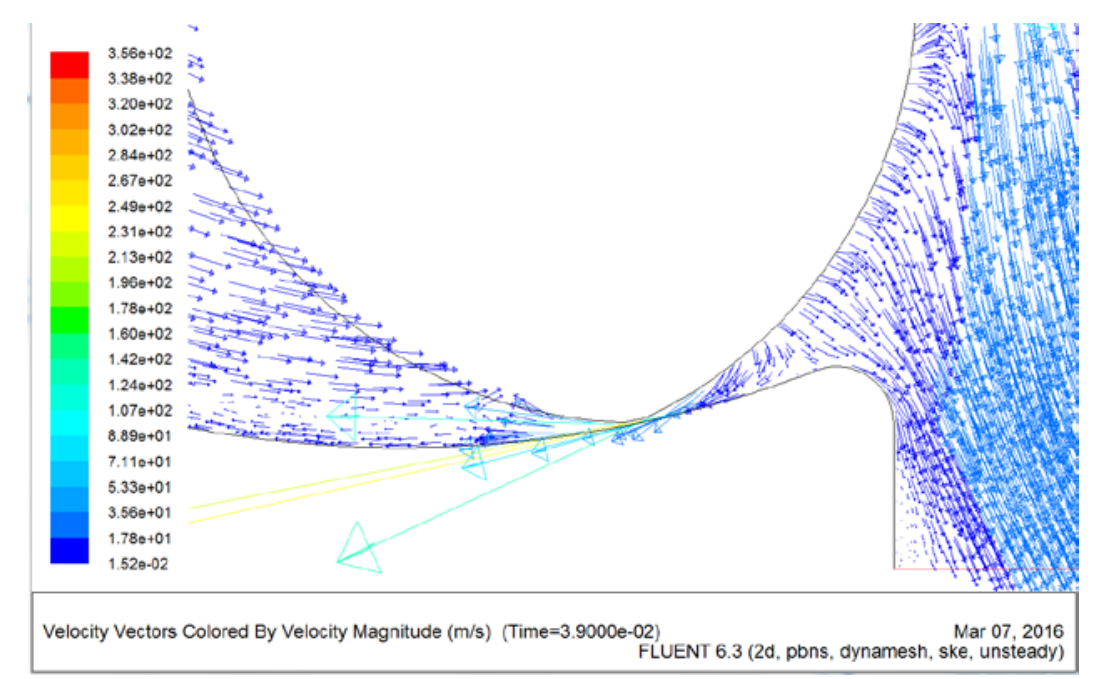

Fig.8 The backflow velocity near the exit of Roots blower with normal gap

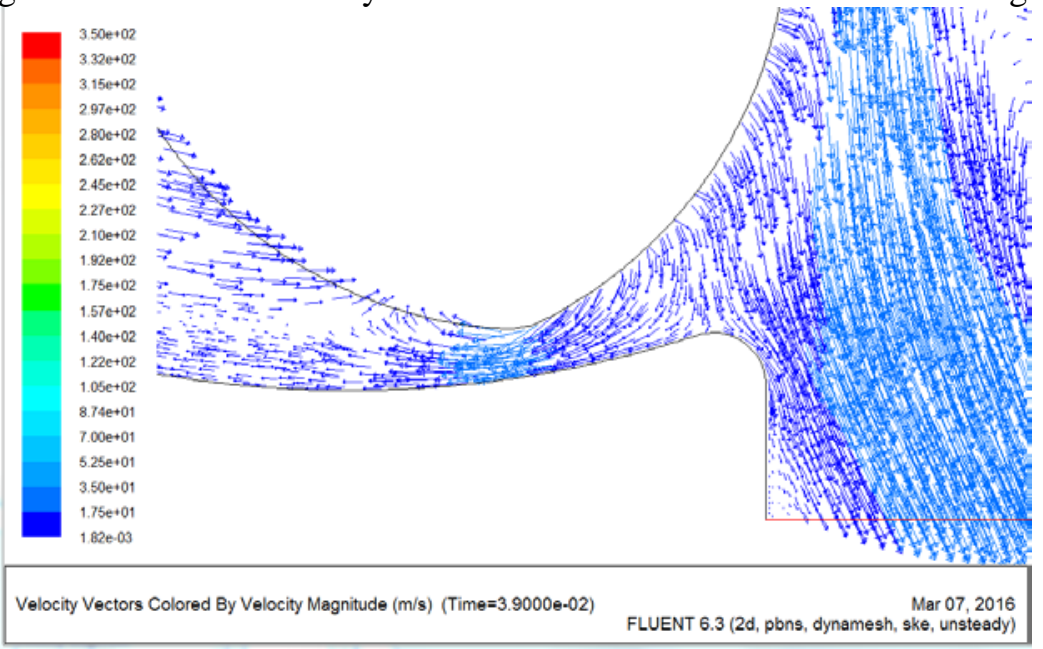

Fig. 9 The backflow velocity near the exit of Roots blower with gradually expanding gap

Before simulation, setting a monitor point nearby the outlet of blower, monitoring the change of flow dynamic pressure. As shown in Fig. 10, the maximum value of the dynamic pressure of the blower with gradually expanding gap declines by $42 \%$ than that of blower with normal gap, and the fluctuation range declines by $62 \%$. The effects of reducing backflow impact and noise are obviously.

\section{Conclusions}

The backflow equation of the gradually expanding gap is established. The optimal value of the gap is solved by the numerical method. And the performances of the blower with the solved parameters are simulated and verified. The results show that the air pulsation and noise can be reduced effectively by using the gradually expanding gap. In addition, the numerical method is used to solve the optimal value of the gradually expanding gap, which provides a theoretical basis for the engineering design.

\section{Acknowledgement}

In this paper, the research was sponsored by the National Natural Science Foundation of China (Project No. 51275092 and No. 51375013) and Fujian Provincial Research and Development Platform for Industrial Robotic Fundamental Components Technology (2014H2004). 


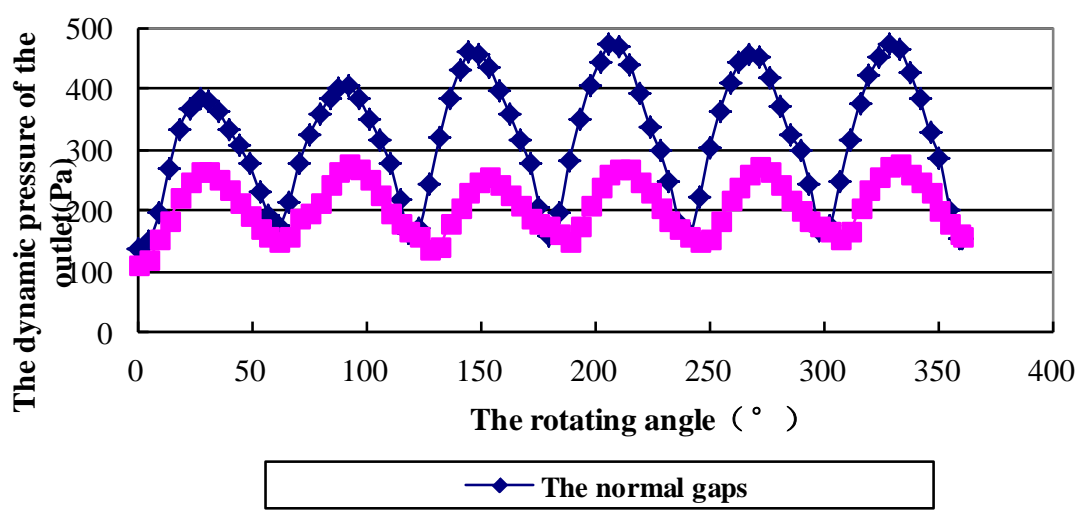

Fig.10. The dynamic pressure of the outlet

\section{Reference}

[1] Su Chunmo. Roots blower and its use [M]. South industrial university press. 1999.

[2] Xiong Weide. The design and manufacture of integrated low noise blower [J]. The research and application of machinery, 2014, 27 (2): 132-135.

[3] Li Zhibao. Roots superchargers for gas pulsation and reduction methods of research [D]. Central south university master's degree thesis. 2014.5.

[4] Wang Zhong, Chen Changzheng, Kong Xiangjun. Isolation system design and noise control of Roots blower room [J]. Advanced materials research. 2014: 971 - 973.

[5] Fu Ming. Circular leaf blower noise reduction of gradually expanding gap [J]. Wind turbine technology, 2012, (1).

[6] Liu Yuanquan, Zhang Baohua. Fluid mechanics [M]. Peking university press, 2013. 9.

[7] He Zhicheng. The principle of chemical engineering (Second edition) [M]. Chinese medical science and technology press. 2009. 8.

[8] Chen Li. Fluid mechanics and pump and fan [M]. Higher education press. 2007. 10.

[9] Yao Ligang, et al. A new blower rotor [P]. The invention patent (2012101458179), 2015. 5. 20.

[10] Long Tianyu, Su Yaxin, et al. Computational fluid mechanics [M]. Chongqing university press, 2002. 3.

[11] Zhang Qiang. Aeroacoustics [M]. Defense industry press. 2012. 9. 